Supplement of

\title{
Use of GNSS SNR data to retrieve soil moisture and vegetation variables over a wheat crop
}

\section{S. Zhang et al.}

Correspondence to: J.-C. Calvet (jean-christophe.calvet@meteo.fr)

Locations of the GPS specular reflection points

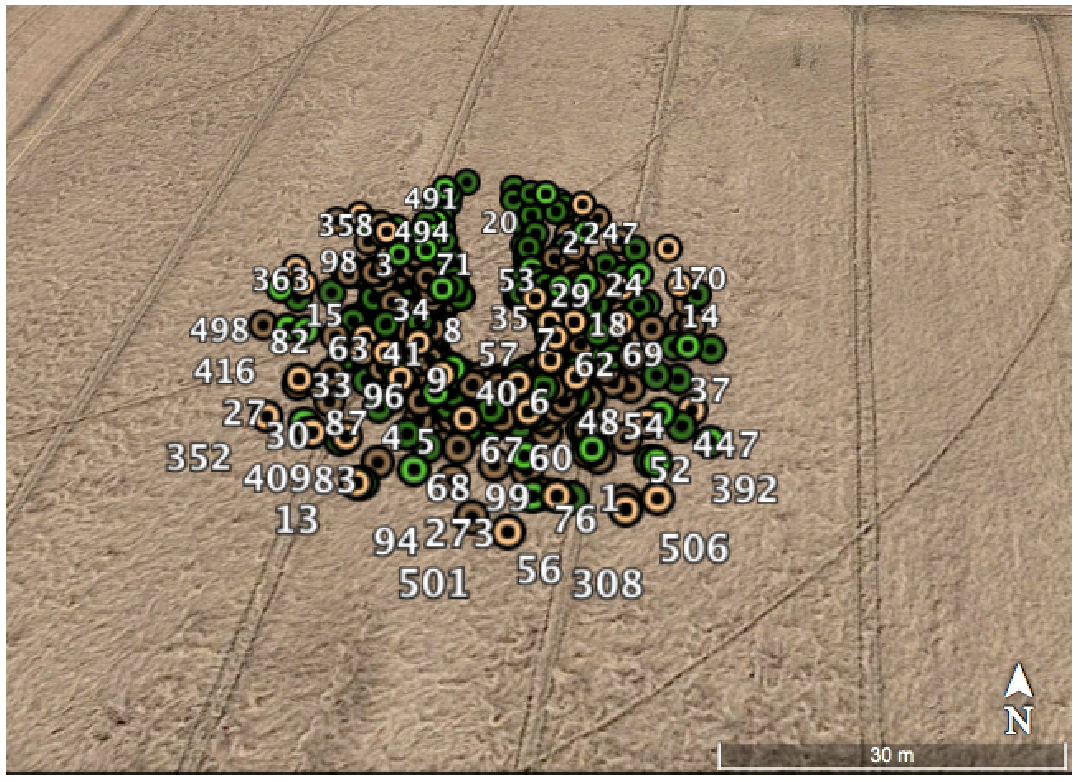

Figure S1 - Locations of the GPS specular reflection points for a GPS receiver on the Lamasquère measurement site. This simulations are done on 21 January 2015. Satellite elevation angles range from 5 to 20 degrees. 


\section{SNR data}

At the Lamasquère site, data from GPS satellites should in theory be received twice per day. However, in practice, some of the satellites were only received once per day.

Figure S2 shows two typical satellite SNR time series for one day (21 January 2015). For GPS01 (top figure), only one ascending track (from low elevation to high elevation) and one descending track (from high elevation to low elevation) were recorded. For GPS18 (bottom figure), there were two ascending tracks and two descending tracks. The observation area (i.e. the reflecting surface) for the ascending track differed from the area seen by the descending track. Thus, we separated the ascending data from data of the descending satellite tracks. For GPS01, we obtained two time series (ascending and descending), and for GPS18 we obtained four time series. Furthermore, GPS01 reached high elevation angles (its maximum elevation angle was about $77^{\circ}$ ), making its elevation angle change faster than that of GPS18 (its maximum elevation angles were about $45^{\circ}$ and $29^{\circ}$ ). Because these differences in maximum satellite elevation angle and elevation angle change rate substantially affected the period of the SNR data, we only used the satellite tracks with at least $40^{\circ}$ maximum elevation for retrieving vegetation height. In the case of Fig. S2, this means that we only used the GPS01 track and the morning GPS18 track. The evening GPS18 track was discarded. Then, within selected tracks, only a valid segment SNR data for elevation angles between $5^{\circ}$ and $20^{\circ}$ were used.

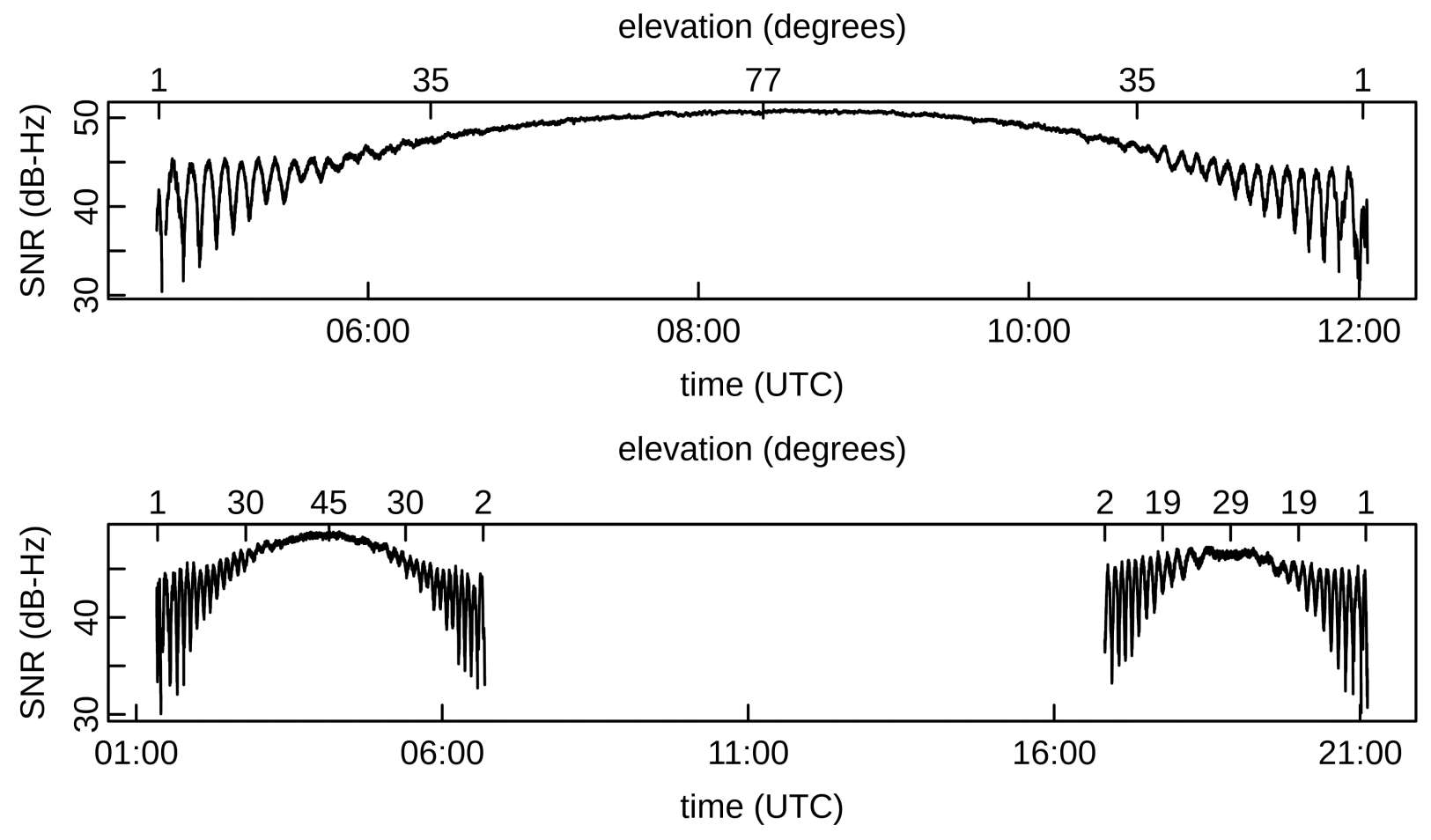

Figure S2 - Recorded S1C SNR data at Lamasquère for (top) GPS01 and (bottom) GPS18, on 21 January 2015. 


\section{Wavelet Analysis}

The WaveletComp $\mathrm{R}$ package analyzes the period structure using the "mother" Morlet wavelet:

$$
\psi(t)=\pi^{-1 / 4} e^{i \omega t} e^{-t^{2} / 2}
$$

The angular frequency $\omega$ is set to 6, as recommended by Torrence and Compo (1998). The Morlet wavelet transform of the multipath SNR time series $\left(\mathrm{SNR}_{\mathrm{mpi}}\right)$ is defined as the convolution of the series with a set of "wavelet daughters" generated by the mother wavelet by translation in time by $\tau$ and scaling by $s$ :

$$
\operatorname{Wave}(\tau, s)=\sum_{t} S N R_{m p i} \frac{1}{\sqrt{s}} \psi^{*}\left(\frac{t-\tau}{s}\right)
$$

where $(*)$ denotes the complex conjugate. The localized estimates of the particular daughter wavelet in the time domain is determined by the localizing time parameter $\tau$ being shifted by a time increment of $\mathrm{d} t$ depending on the sampling interval. The wavelet transform is computed for a wavelet scale $(s)$ set of interest, which is a fractional power of 2 ,

$$
s_{j}=s_{\min } 2^{j d j}, j=0,1, \ldots, J
$$

The minimum (maximum) scale is fixed via the choice of the minimum (maximum) period interest depending on the possible relative antenna height change through the conversion factor $6 /(2 \pi)$. In this study they were set as $128 \mathrm{~s}$ and $1024 \mathrm{~s}$, respectively. The wavelet transform can be separated into real part and imaginary part, thus providing information on both local amplitude and instantaneous phase of any periodic process across time. The local power of any periodic component of the time series under investigation is

$$
\operatorname{Power}(\tau, s)=\frac{1}{s}|\operatorname{Wave}(\tau, s)|^{2}
$$

which is called as the wavelet power spectrum.

\section{References}

Torrence, C., and G. P. Compo, 1998: A practical guide to wavelet analysis, Bulletin of the American Meteorological Society, 79(1), 61-78.

Roesch, A., H. Schmidbauer, and M. A. Roesch, 2014: 'WaveletComp' package. 


\section{Data sorting for vegetation height retrieval}

The wavelet analysis is able to detect situations during which vegetation height retrieval is not possible. This is illustrated in Fig. S3 for SNR data (black line in Fig. S3a) acquired on 15 June 2015. There are two peaks in the average power spectrum as shown in Fig. S3b. Furthermore, the maximum average power is only 0.54 which is significantly smaller than the maximum average power of 1.4 observed on 21 January 2015 (see Fig. 1b). In Fig. S3a, the reconstructed SNR data (red line) corresponding to the $T_{d}(322.5 \mathrm{~s}$ in this case) cannot fit the SNR data as well as in Fig. 1a. The SNR pattern is also clearly more noisy, with smaller amplitudes and less clear pattern in Fig. S3a than in Fig. 1a. A possible cause is the more inhomogeneous reflecting surface after the lodging event. Fig. S4 gives the dominant period $\left(T_{d}\right)$ time series derived from GPS01 ascending tracks from 16 January 2015 to 15 July 2015 and the green crosses indicate poor quality data, which were excluded from the $T_{d}$ time series.

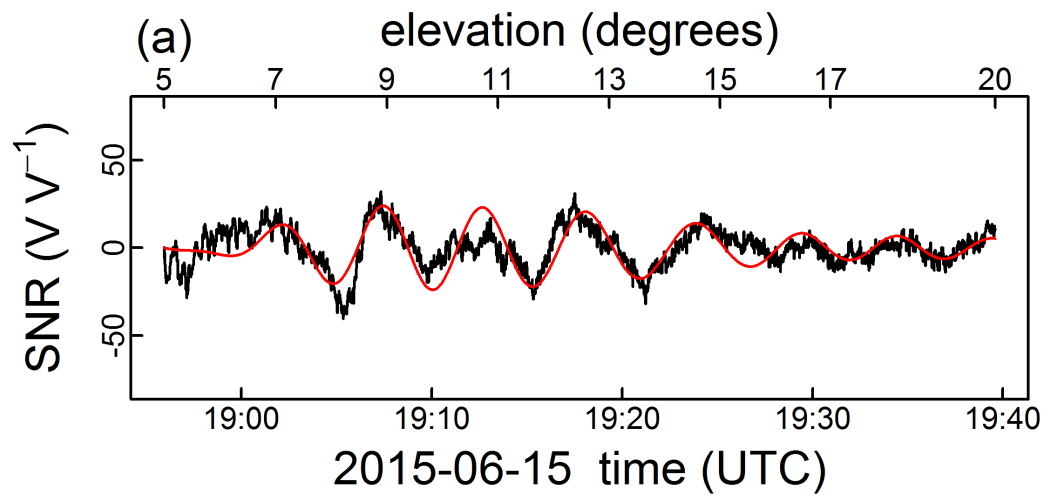

(b)

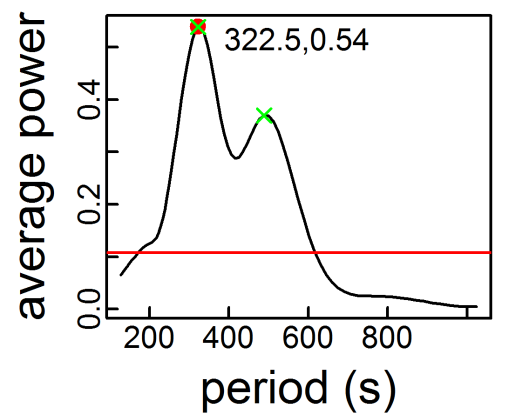

Figure S3 - Example of an unusable track data set: (a) SNR data (black line) and (b) average power spectrum for the ascending track of GPS01 on 15 June 2015. The red line in (a) is the reconstructed SNR data by the daughter wavelet corresponding to the maximum peak period (322.5 s) in (b). The green crosses in (b) show there are two peak periods in this track data, indicating bad quality, unusable data. 

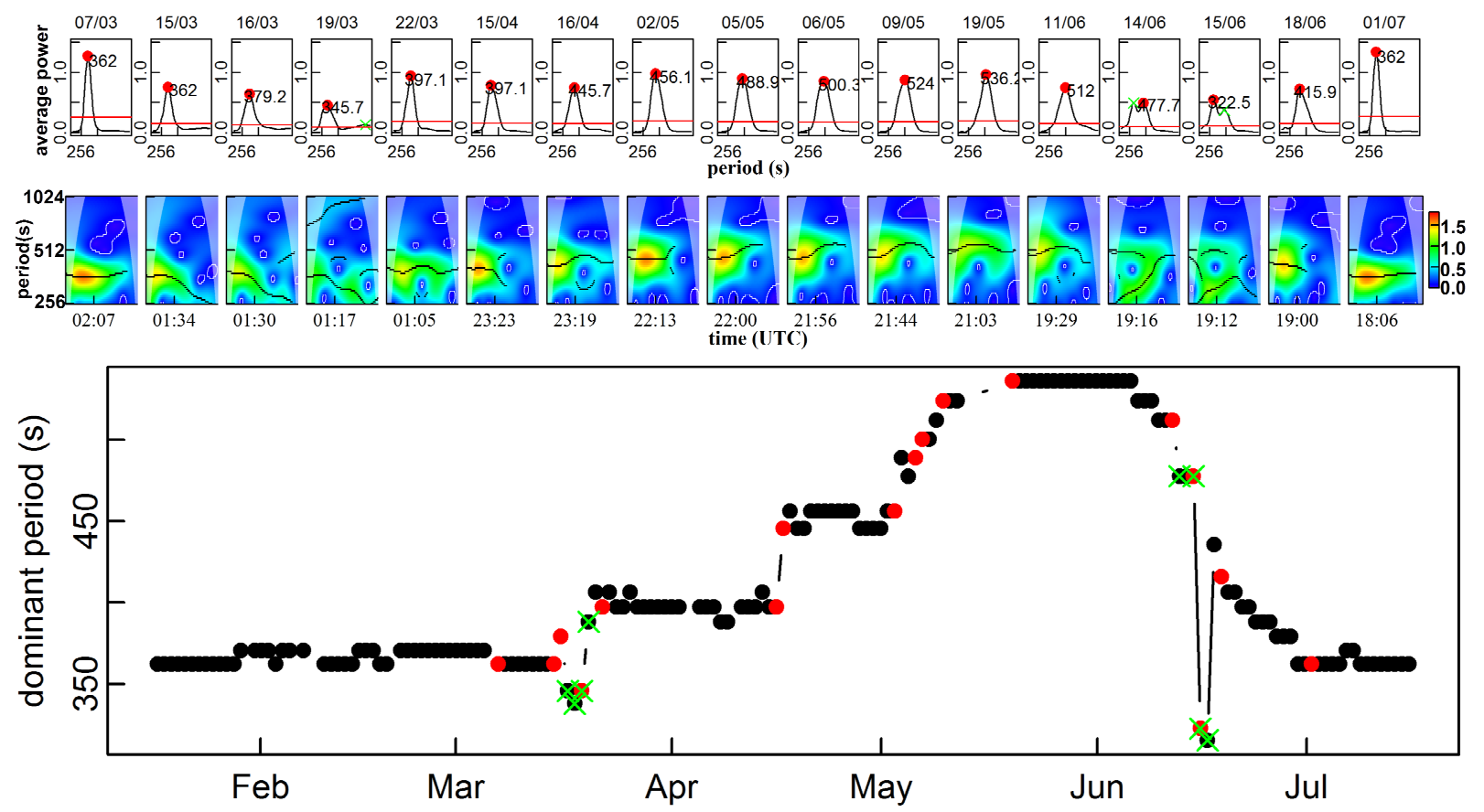

Figure S4 - SNR dominant period $\left(T_{d}\right)$ time series (black dots in the bottom sub-figure) derived from the GPS01 ascending tracks, with the green crosses indicate more than one peak are recognized as bad quality data, from 16 January to 15 July, 2015. And (top) the average power spectrums and (middle) power spectrums on the selected days (red dots in the bottom sub-figure) are also shown. 


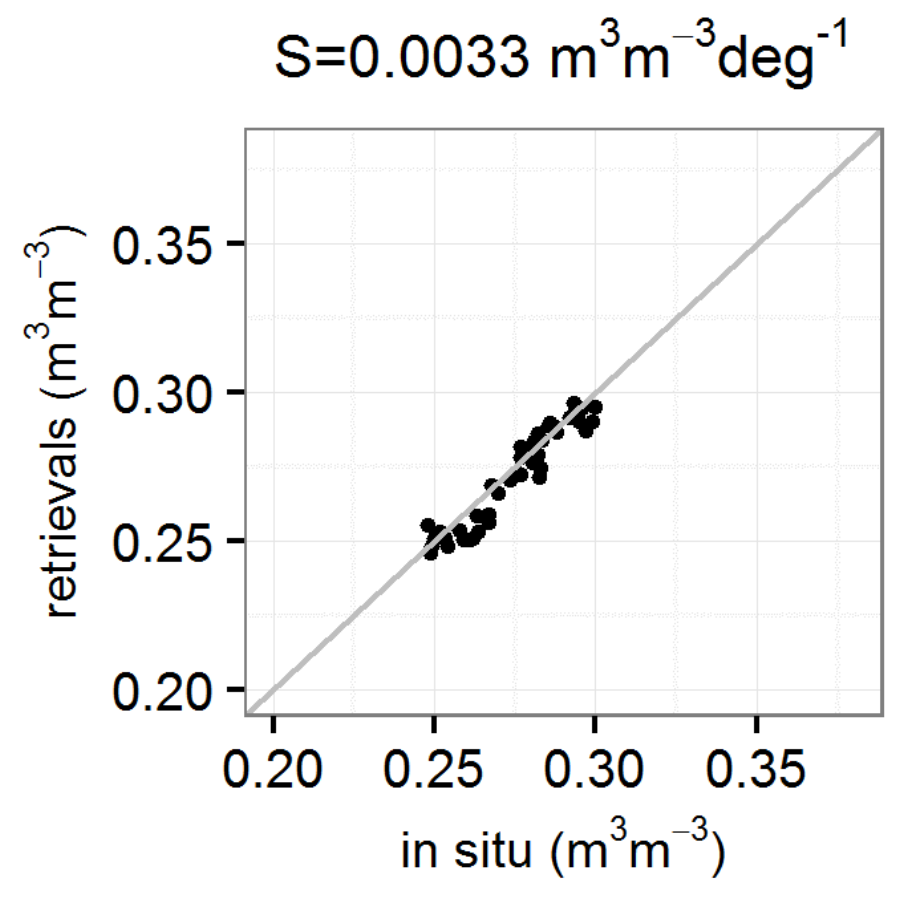

Figure S5 - Volumetric soil moisture (VSM) GPS retrievals versus in situ VSM observations $\left(\mathrm{m}^{3} \mathrm{~m}^{-3}\right)$ from 16 January to 5 March 2015 , with fitted slope $=0.0033 \mathrm{~m}^{3} \mathrm{~m}^{-3} \mathrm{deg}^{1}$ for satellite tracks whose phase presents a linear correlation with in situ soil moisture higher than 0.9 . This occurred for the ascending tracks of GPS 13, 21, 24, 30 and for the descending tracks of GPS $05,09,10,15$, and 23 . 


\section{VSM and multipath phase $\left(\varphi_{m p i}\right)$}

We compared the multipath phase time series with in situ VSM observations and ISBA VSM simulations from 16 January to 15 July (the entire wheat growth cycle). It is separated into two time periods, from 16 January to 5 March (with no significant vegetation effects, $A_{\text {norm }}>$ 0.78 ) and from 6 March to 15 July (with big vegetation effects, $A_{\text {norm }}<0.78$ ). There is a good linear correlation ( 0.92 , with 47 valid observations) between multipath phase and in situ VSM observations from 16 January to 5 March when there is no significant vegetation effects. During this time period, the same results are obtained with or without unwrapping of the phase. The correlation coefficient is 0.86 (with 43 valid data) between multipath phase and ISBA simulated VSM during the same time period. But after 5 March, multipath phase is not related to either observed or simulated VSM. We compared the multipath phase with the observed and simulated VSM, with or without unwrapping. The correlation coefficient is 0.48 (with 51 valid data) between the unwrapped multipath phase and the observed VSM, and the correlation coefficient is -0.10 (with 114 valid data) between the unwrapped multipath phase and the simulated VSM. Figure S6 also shows the impact of not unwrapping the phase.

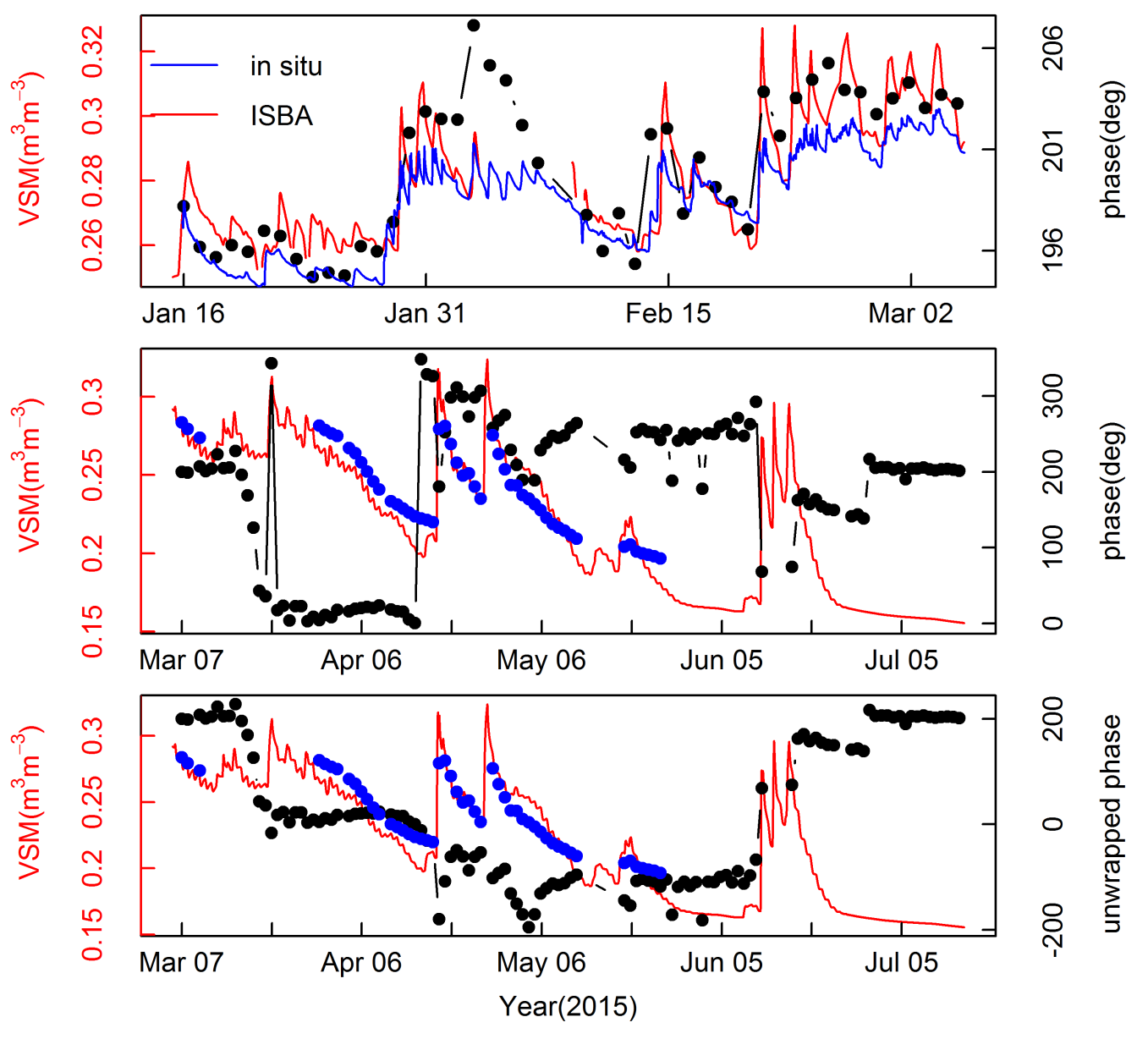

Figure S6 - Example of a track data set (descending tracks from GPS10): (top) from 16 January to 5 March, with no significant vegetation effects; (middle and bottom) from 6 March to 15 July, with significant vegetation effects. In the top and middle figures, multipath phases (black dots) are compared with in situ VSM measurements at $5 \mathrm{~cm}$ (blue line or dots) and ISBA simulations (red line). In the bottom figure, unwrapped multipath phases (black dots) are used to compare with in situ and simulated VSM. 


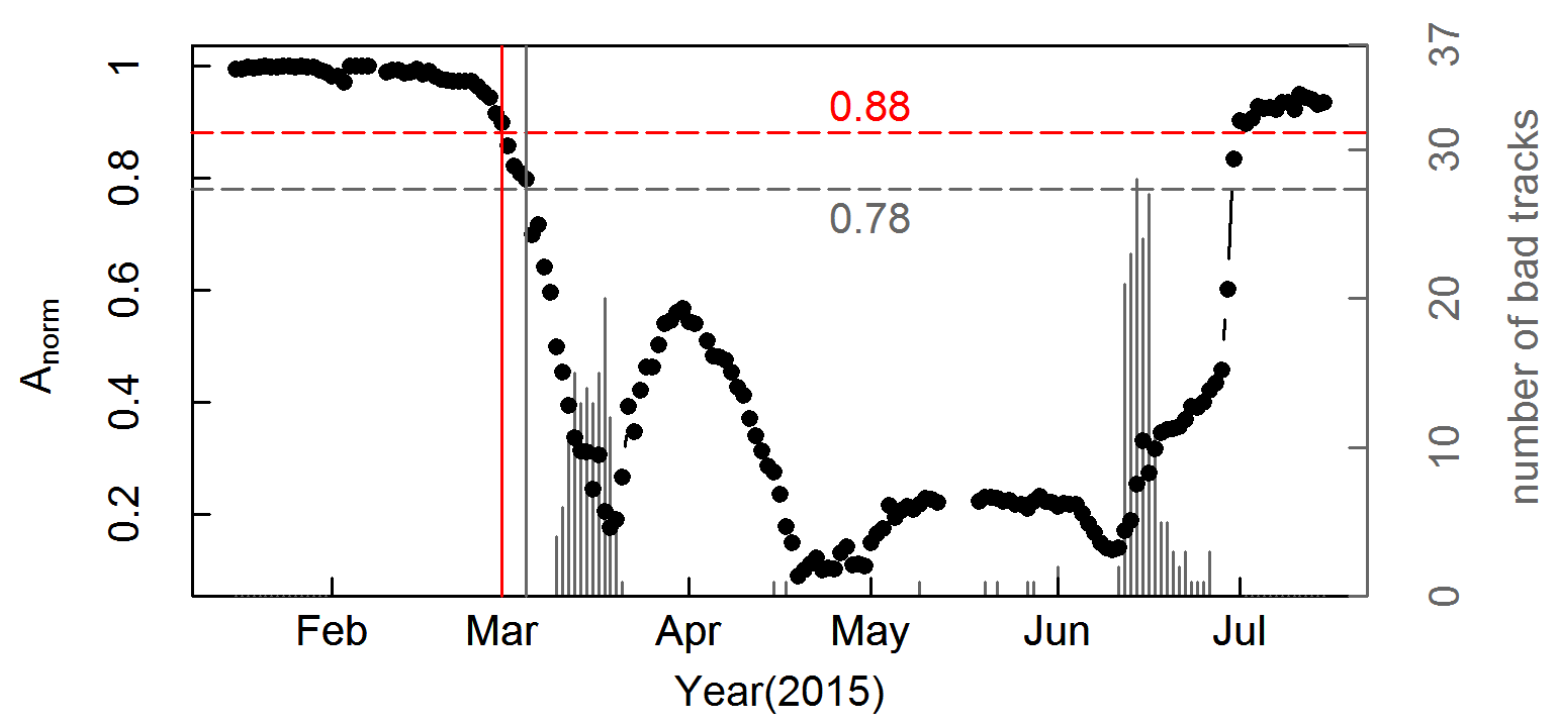

Figure S7 - Normalized amplitude $\left(A_{\text {norm }}\right)$ time series (black dots) and probability distribution (grey bars) of low quality tracks among all available satellite tracks on a daily basis from 16 January to 15 July 2015 . The empirical $A_{\text {norm }}$ threshold $(0.78)$ is shown by the grey dashed line, and the soil moisture can be retrieved from 16 January to 5 March 2015 depending on it. Our field estimated $A_{\text {norm }}$ threshold (0.88) depending on the $A_{\text {norm }}$ on 1 July is shown by the red dashed line, and it indicates the soil moisture can be retrieved from 16 January to 1 March 2015. 


\section{Effect of wheat growth on estimating $A_{\text {norm }}$}

Figure S8a shows SNR data acquired on 1 May 2015. Its maximum average power is close to 1 (Fig. S8b), but its $A_{\text {norm }}$ is only 0.15 (Fig. S7). Comparing Fig. S8a and Fig. S3a, it can be observed that the signal amplitude is larger on 1 May than that on 15 June. But $A_{\text {norm }}(0.15)$ on 1 May is even smaller than the $A_{\text {norm }}(0.33)$ on 15 June. Because the hypothesis of a constant effective antenna height is not valid, it is likely that $A_{\text {norm }}$ was underestimated on 1 May.

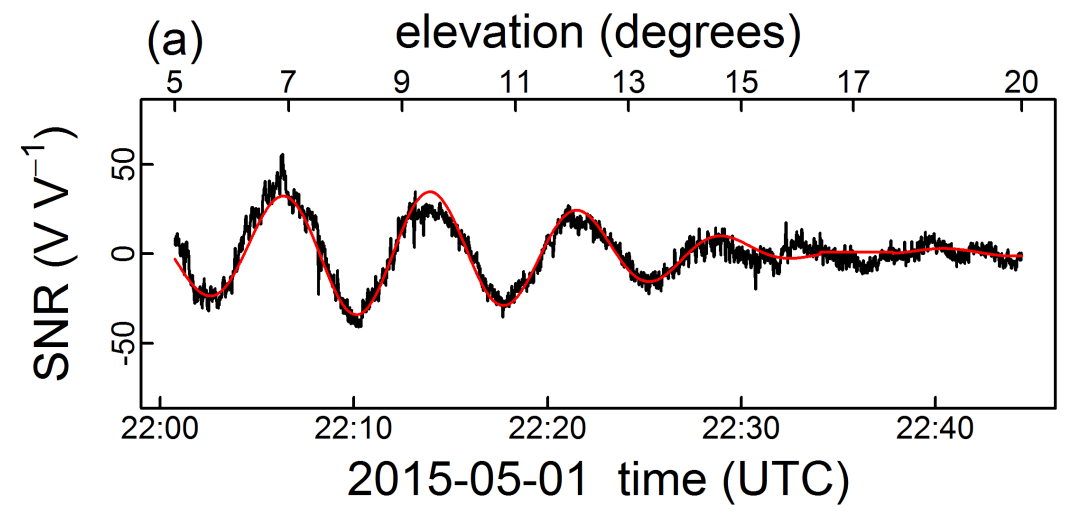

(b)

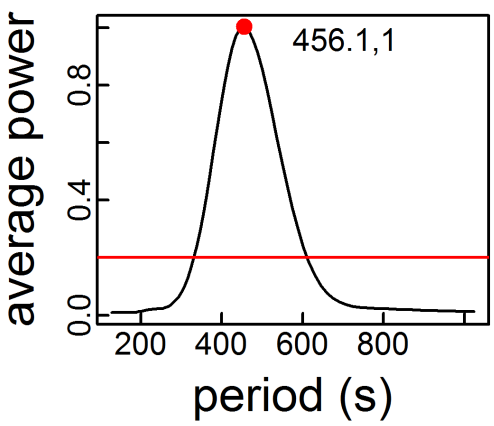

Figure S8 - Example of a track data set: (a) SNR data (black line) and (b) average power spectrum for the ascending track of GPS01 on 1 May 2015. The red line in (a) is the reconstructed SNR data by the daughter wavelet corresponding to the peak period $(456.1 \mathrm{~s})$ in (b).

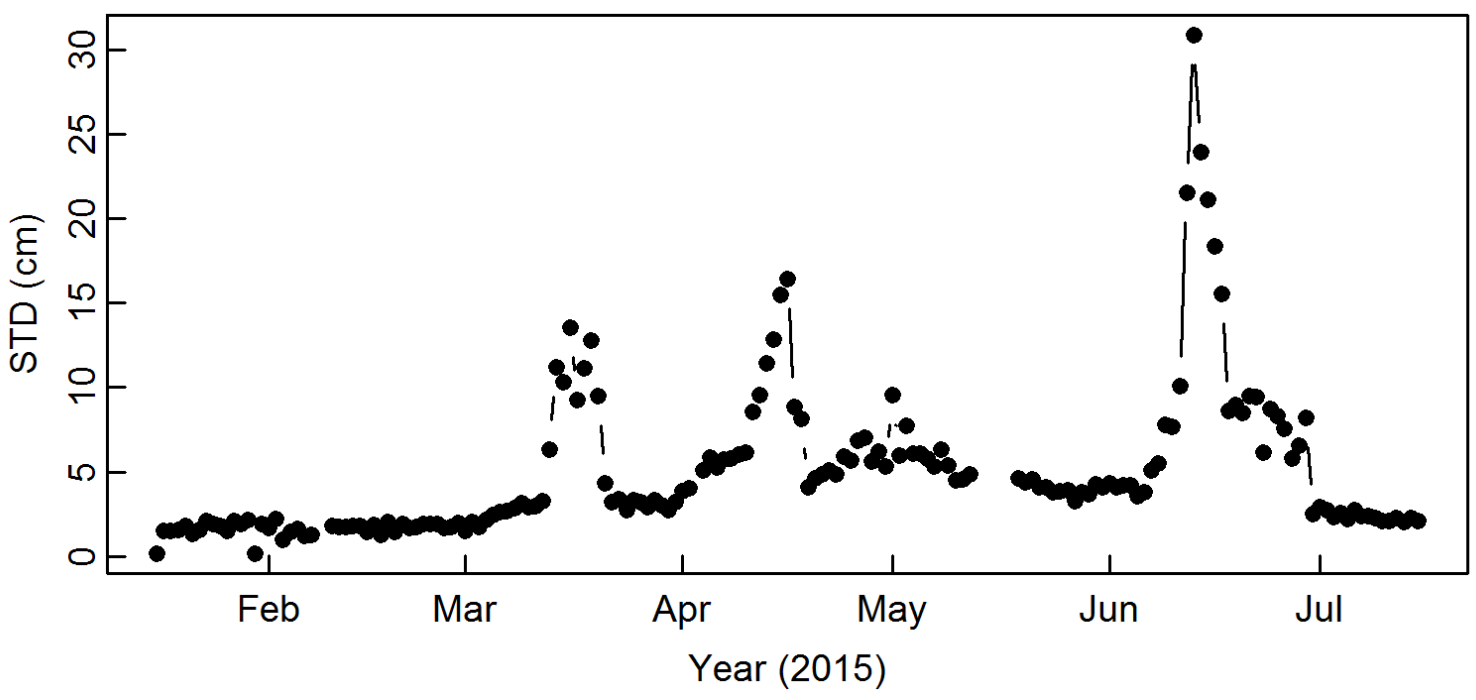

Figure S9 - The daily standard deviation (STD) score of the retrieved vegetation height from all available satellite tracks from 16 January to 15 July 2015. 

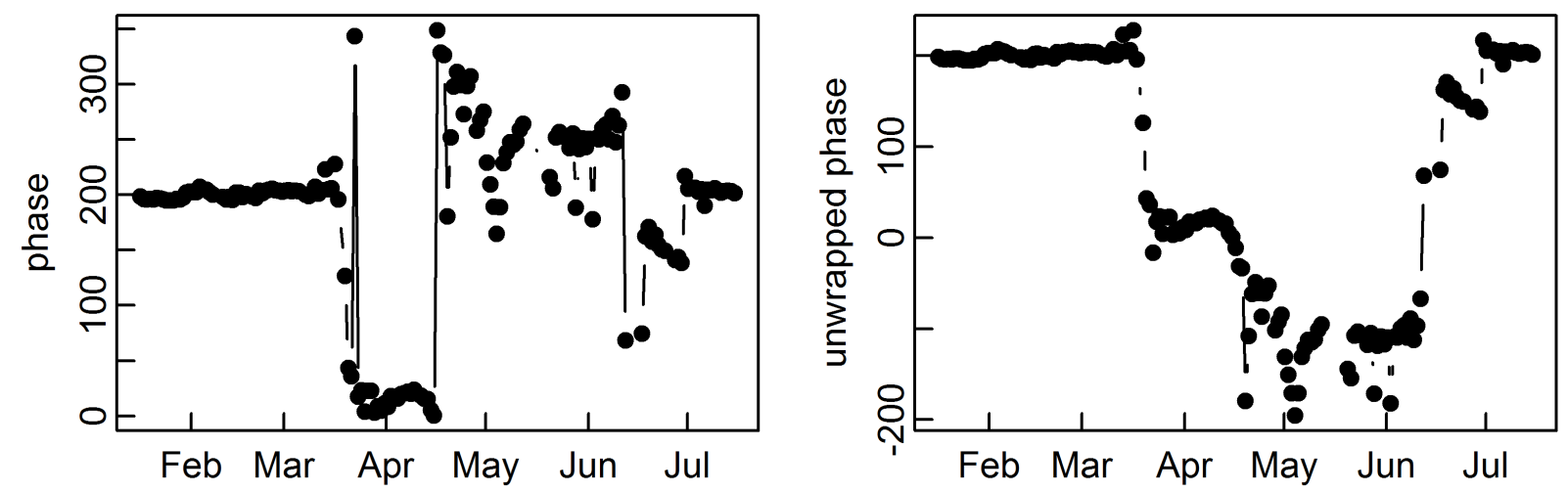

Figure S10 - Example of a track data set: (left) multipath phase $\left(\varphi_{m p i}\right.$, in degrees) in Eq. (5) and (right) unwrapped multipath phase (in degrees) for the descending tracks of GPS10 from 16 January to 15 July 2015. 


\section{GDD (growing degree days) model}

Temperatures at 2 meter from a large scale simulations made over France with a spatial resolution of $8 \mathrm{~km}$ by $8 \mathrm{~km}$ were extracted in order to build the growing degree days (GDD) model for Lamasquère site. The reference data was between year 2009 and 2010, it was sown on 1 October 2009, and the tillering, flowering and ripening were on 26 March, 9 June and 12 June 2010, respectively. $T_{G D D}$ is calculated as the accumulation of daily mean temperatures $\left(T_{\text {mean }}\right), T_{\text {mean }}$ is calculated in the following way:

$T_{\text {mean }}=\frac{T_{\max }+T_{\min }}{2}-T_{\text {base }}$

based on the daily minimum $\left(T_{\min }\right)$ and maximum $\left(T_{\max }\right)$ temperatures. $T_{\text {mean }}$ is further forced to range between $0^{\circ} \mathrm{C}$ and $35^{\circ} \mathrm{C}$. The base temperature $\left(T_{\text {base }}\right)$ used here for winter wheat is $0^{\circ} \mathrm{C}$ and the starting date for the accumulated temperature is from 1 October 2009, the accumulated temperature $\left(T_{G D D}\right)$ is calculated as

$T_{G D D}=\sum T_{\text {mean }}$

This GDD model was applied to year 2015 for our study, according to the $T_{G D D}$ in the GDD model, we obtained the following dates for tillering, flowering, and ripening: $12 \mathrm{March}, 31$ May, and 3 June 2015, respectively.
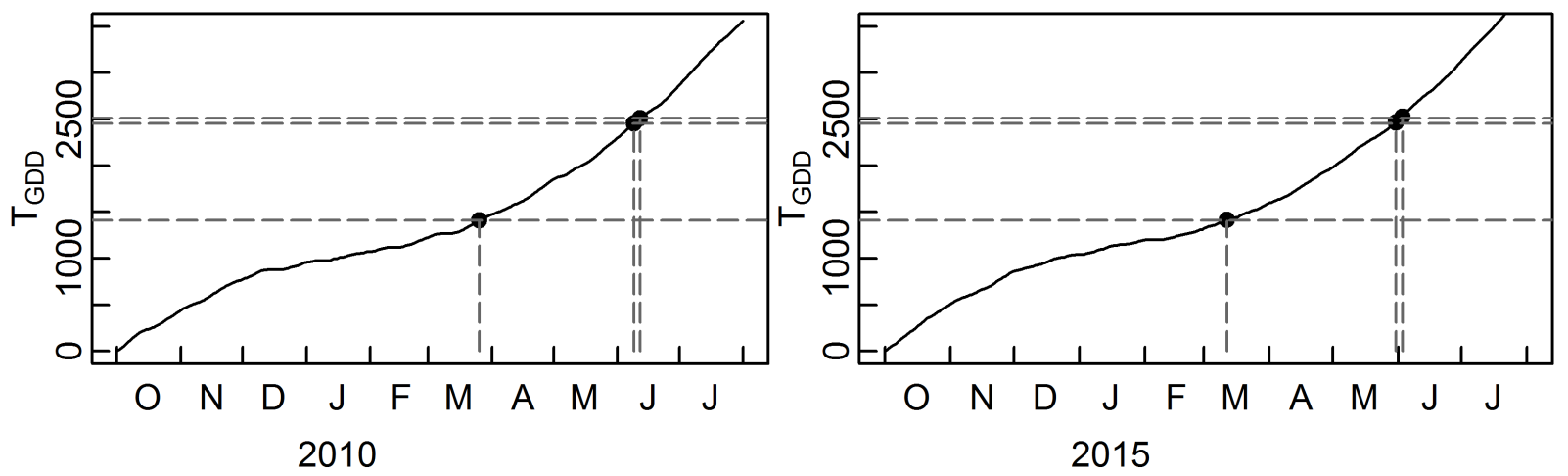

Figure S11 - (left) The growing degree days (GDD) model build in 2010 for winter wheat at Lamasquère and (right) GDD model build in 2015 at the same site. According to the $T_{G D D}$ $\left({ }^{\circ} \mathrm{C}\right)$ on the tillering, flowering and ripening in 2010 , the corresponding phenological stage dates in 2015 are estimated.

\section{References}

Betbeder, J., Fieuzal, R., Philippets, Y., Ferro-Famil, L., and Baup, F. (2016) Contribution of multitemporal polarimetric synthetic aperture radar data for monitoring winter wheat and rapeseed crops, J. Appl. Remote Sens. 10(2), 026020, doi: 10.1117/1.JRS.10.026020, 2016.

Duveiller, G., Weiss, M., Baret, F., \& Defourny, P. (2011). Retrieving wheat green area index during the growing season from optical time series measurements based on neural network radiative transfer inversion. Remote Sensing of Environment, 115(3), 887-896. 


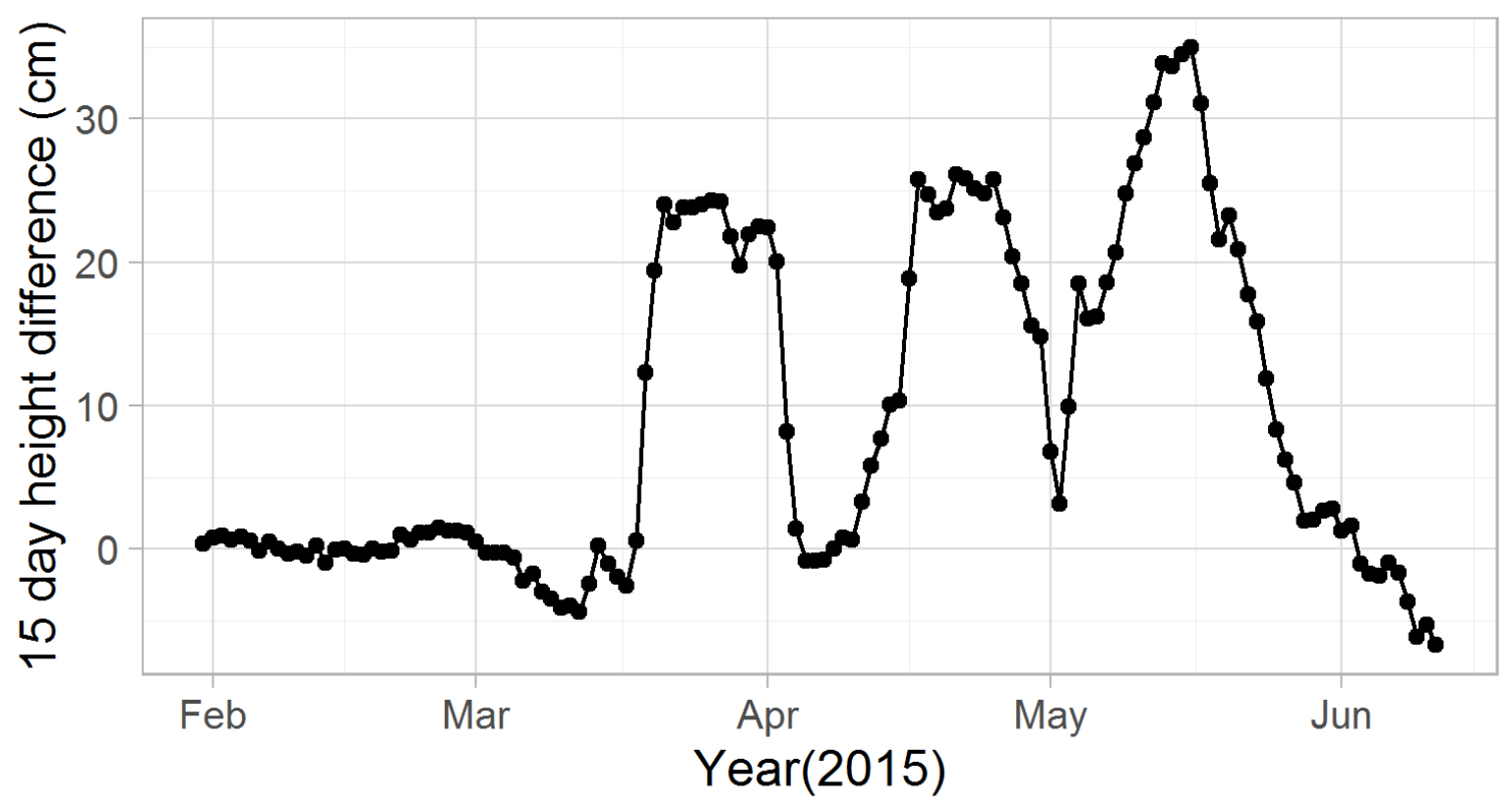

Figure S12 - Retrieved vegetation height difference $(\mathrm{cm})$ with the value retrieved 15 days before, from 31 January to 11 June 2015. 